\title{
On stability of the general linear equation
}

\author{
Anna Bahyrycz and Jolanta OlKo
}

\begin{abstract}
We prove, using the fixed point approach, some stability results for the general linear functional equation. Namely we obtain sufficient conditions for the stability of a wide class of functional equations and control functions. Our results generalize a lot of the well known and recent outcomes concerning stability. In some examples we indicate how our method may be used to check if the particular functional equation is stable and we discuss the optimality of obtained bounding constants.
\end{abstract}

Mathematics Subject Classification. Primary 39B82; Secondary 39B52.

Keywords. Hyers-Ulam stability, linear functional equation, fixed point theorem.

\section{Introduction}

The theory of stability of functional equations started with Hyers' answer to the famous question of Ulam concerning the stability of homomorphisms in metric groups (cf. [9]). Since then many authors have studied this subject dealing with a lot of functional equations, for example:

$$
\begin{aligned}
& \text { p-Wright equation } f(p x+(1-p) y)+f((1-p) x+p y)=f(x)+f(y) ; \\
& \text { linear equation } f(a x+b y)=A f(x)+B f(y) \\
& \text { quadratic equation } f(x+y)+f(x-y)=2 f(x)+2 f(y) \\
& \text { Fréchet's equation } f(x+y+z)+f(x)+f(y)+f(z) \\
&=f(x+y)+f(x+z)+f(y+z),
\end{aligned}
$$

where $a, b, A, B, p$ are given (cf., e.g., [1,2,5,7,8,10,11,13-17]).

We deal with the general linear equation, in the class of functions mapping a linear space $X$ into a normed space $Y$ (both over the field $\mathbb{F} \in\{\mathbb{R}, \mathbb{C}\}$ ), namely 


$$
\sum_{i=1}^{m} A_{i} f\left(\sum_{j=1}^{n} a_{i j} x_{j}\right)+A=0,
$$

where $A, a_{i j} \in \mathbb{F}, A_{i} \in \mathbb{F} \backslash\{0\}, i \in\{1, \ldots, m\}, j \in\{1, \ldots, n\}$.

One of the ways of proving stability is the method of fixed points which is the most popular tool. Analyzing proofs of stability in particular cases of the functional equation (1.1), among others the above-mentioned (see [3,4]), we have found sufficient conditions for its stability.

What is more interesting, we present some examples how our criterion may be used as a tool to check the stability of particular functional equations of the linear type. Moreover, we discuss the optimality of obtained bounding constants in particular cases.

Some similar ideas can be found in [6], where the general method for proving stability is described. Our considerations based on the fixed point theorem lead to a simpler procedure and sufficient conditions which are easier to check.

\section{Statement of the main result and applications}

In this section we state the main result and we present its applications. The proof of the theorem will be given in the last section. The main theorem of this paper provides a criterion for the stability of the equation (1.1). It implies lots of the well known and recent results concerning particular cases of this functional equation. We describe a method of determining whether a functional equation of linear type is stable.

Our test method for stability of the equation is based on the use of the fixed point theorem. In proofs of numerous theorems concerning stability an appropriate substitution is used to obtain a contraction operator. The fixed point of the operator is a solution of the equation which is close to a given function.

Namely, considering a function $g$ satisfying approximately equation (1.1) we look for a substitution $x_{j}=c_{j} x, c_{j} \in \mathbb{F}, j \in\{1, \ldots, n\}$ such that

$$
\sum_{i=1}^{m} A_{i} g\left(\sum_{j=1}^{n} a_{i j} c_{j} x\right)+A=g(x)-\mathcal{T} g(x), \quad x \in X,
$$

where $\mathcal{T}$ is a proper operator. For this purpose, the solution $c_{1}, \ldots, c_{n} \in \mathbb{F}$ to the system consisting of at least one equation

$$
\sum_{j=1}^{n} a_{i j} c_{j}=1, \quad i \in\{1, \ldots, m\},
$$

should be found [see condition (i)]. In the following theorem additional conditions implying stability are given. 
In the sequel $\mathbb{R}_{+}=[0,+\infty)$. A sum of numbers over an empty set is defined to be zero.

Theorem 2.1. Assume that $A=0$ or $\left(A \neq 0\right.$ and $\left.\sum_{i=1}^{m} A_{i} \neq 0\right)$. Let $Y$ be a Banach space, $g: X \rightarrow Y, \theta: X^{n} \rightarrow \mathbb{R}_{+}$fulfill

$$
\left\|\sum_{i=1}^{m} A_{i} g\left(\sum_{j=1}^{n} a_{i j} x_{j}\right)+A\right\| \leq \theta\left(x_{1}, \ldots, x_{n}\right), \quad x_{1}, \ldots, x_{n} \in X .
$$

Assume that there exist $\emptyset \neq I \subset\{1, \ldots, m\}, c_{1}, \ldots, c_{n} \in \mathbb{F}$ and $\omega_{1}, \ldots, \omega_{n} \in$ $[0,+\infty)$ such that

(i) $\sum_{j=1}^{n} a_{i j} c_{j}=1, i \in I$,

(ii) $\sum_{i \notin I}\left|A_{i}\right| \omega_{i}<\left|\sum_{i \in I} A_{i}\right|$,

(iii) $\theta\left(\sum_{j=1}^{n} a_{i j} c_{j}\left(x_{1}, \ldots, x_{n}\right)\right) \leq \omega_{i} \theta\left(x_{1}, \ldots, x_{n}\right) \quad i \notin I, x_{1}, \ldots, x_{n} \in X$.

Then there exists a unique solution $G: X \rightarrow Y$ of (1.1) such that

$$
\|g(x)-G(x)\| \leq \frac{\theta\left(c_{1} x, \ldots, c_{n} x\right)}{\left|\sum_{i \in I} A_{i}\right|-\sum_{i \notin I}\left|A_{i}\right| \omega_{i}}, \quad x \in X .
$$

Moreover $G$ is a unique solution of (1.1) such that there exists a constant $B \in(0, \infty)$ with

$$
\|g(x)-G(x)\| \leq B \theta\left(c_{1} x, \ldots, c_{n} x\right), \quad x \in X .
$$

Remark 2.2. Observe that if (i) holds with $I=\{1, \ldots, m\}$, then the conditions (ii), (iii) are satisfied. Consequently the equation is stable.

Remark 2.3. Among frequently appearing control functions in (2.1) are the following

1. $\theta_{1}\left(x_{1}, \ldots, x_{n}\right)=C$;

2. $\theta_{2}\left(x_{1}, \ldots, x_{n}\right)=C \sum_{j=1}^{n}\left\|c_{j} x_{j}\right\|^{k_{j}}$

3. $\theta_{3}\left(x_{1}, \ldots, x_{n}\right)=C \max \left\{\left\|c_{j} x_{j}\right\|^{k_{j}}: j \in\{1, \ldots, n\}\right\}$;

4. $\theta_{4}\left(x_{1}, \ldots, x_{n}\right)=C \sqcap_{j=1}^{n}\left\|x_{j}\right\|^{k_{j}}$;

with some $C \in(0,+\infty), k_{j}>0$ and $c_{j} \in \mathbb{F} \backslash\{0\}$.

It is easy to see that for each such function $\theta_{i}$ there exists $\omega: \mathbb{F} \rightarrow \mathbb{R}_{+}$ fulfilling

$$
\theta_{i}\left(\beta x_{1}, \ldots, \beta x_{n}\right) \leq \omega(\beta) \theta_{i}\left(x_{1}, \ldots, x_{n}\right) \quad \beta \in \mathbb{F}, x_{1}, \ldots, x_{n} \in X,
$$

(for example $\omega \equiv 1$ for $\theta_{1}, \omega(c)=\max \left\{|c|^{k_{1}}, \ldots,|c|^{k_{n}}\right\}, c \in \mathbb{F}$ for $\theta_{2}$ ). Consequently the condition (iii) is satisfied with $\omega_{i}=\omega\left(\sum_{j=1}^{n} a_{i j} c_{j}\right)$. 
Our result may be used for proving the stability of different functional equations of the general form (1.1). Below we indicate relevant examples of its applications, to present our method.

\section{1. $p$-Wright equation}

Consider the $p$-Wright equation with $p \in \mathbb{F} \backslash\{0,1\}$

$$
f(p x+(1-p) y)+f((1-p) x+p y)=f(x)+f(y), \quad x, y \in X,
$$

which is a particular case of (1.1), with $m=4, n=2, A_{1}=A_{2}=1, A_{3}=$ $A_{4}=-1, a_{11} a_{22}=p, a_{12}=a_{21}=1-p, a_{31}=a_{42}=1, a_{32}=a_{41}=0$. We look for solutions $c_{1}, c_{2}$ of subsystems of the system of linear equations

$$
\left[\begin{array}{cc}
p & 1-p \\
1-p & p \\
1 & 0 \\
0 & 1
\end{array}\right]\left[\begin{array}{l}
c_{1} \\
c_{2}
\end{array}\right]=\left[\begin{array}{l}
1 \\
1 \\
1 \\
1
\end{array}\right]
$$

which leads to the following two cases

$$
\begin{aligned}
& \text { (1) }\left\{\begin{array}{l}
p c_{1}+(1-p) c_{2}=1 \\
(1-p) c_{1}+p c_{2} \neq 1 \\
c_{1} \neq 1 \\
c_{2} \neq 1
\end{array}\right. \\
& \text { (2) }\left\{\begin{array} { l } 
{ p c _ { 1 } + ( 1 - p ) c _ { 2 } \neq 1 } \\
{ ( 1 - p ) c _ { 1 } + p c _ { 2 } \neq 1 } \\
{ c _ { 1 } = 1 } \\
{ c _ { 2 } = c \neq 1 }
\end{array} \Longleftrightarrow \left\{\begin{array}{l}
c_{1}=c \neq 1 \\
c_{2}=\frac{1-p c}{1-p}
\end{array}\right.\right. \\
&
\end{aligned}
$$

According to Theorem 2.1 applied to these solutions, we obtain estimations in the real case for a particular control function. To shorten the paper we omit the second conclusion of Theorem 2.1 (see condition (2.3)) in the statement of the following results.

Theorem 2.4. Let $\mathbb{F}=\mathbb{R}, k \in(0, \infty), p \in \mathbb{R} \backslash\{0,1\}, c \in \mathbb{R} \backslash\{1\}$. Assume that $g: X \rightarrow Y$ satisfies the inequality $\|g(p x+(1-p) y)+g((1-p) x+p y)-g(x)-g(y)\| \leq\|x\|^{k}+\|y\|^{k}, \quad x, y \in X$. If $\gamma_{1}(c):=\left|(1-p) c+p \frac{1-p c}{1-p}\right|^{k}+|c|^{k}+\left|\frac{1-p c}{1-p}\right|^{k}<1$, then there exists a unique $p$-Wright affine function $G: X \rightarrow Y$ such that

$$
\|g(x)-G(x)\| \leq \frac{\left(|c|^{k}+\left|\frac{1-p c}{1-p}\right|^{k}\right)\|x\|^{k}}{1-\gamma_{1}(c)}, \quad x \in X .
$$


If $\gamma_{2}(c):=|p+(1-p) c|^{k}+|(1-p)+p c|^{k}+|c|^{k}<1$, then there exists a unique $p$-Wright function $G: X \rightarrow Y$ such that

$$
\|g(x)-G(x)\| \leq \frac{\left(1+|c|^{k}\right)\|x\|^{k}}{1-\gamma_{2}(c)}, \quad x \in X .
$$

An analysis of the conditional minima of the above obtained bounding functions in the case $k=2$ ensures the stability of this equation in this case. The detailed verification of the following corollary is left to the reader.

Corollary 2.5 (The case $k=2$ ). Let $p \in \mathbb{R} \backslash\{0,1\}, g: X \rightarrow Y$ satisfy $\|g(p x+(1-p) y)+g((1-p) x+p y)-g(x)-g(y)\| \leq\|x\|^{2}+\|y\|^{2}, \quad x, y \in X$.

Then there exists a unique $p$-Wright affine function $G: X \rightarrow Y$ such that

$$
\|g(x)-G(x)\| \leq M\|x\|^{2}, \quad x \in X,
$$

where

$$
M= \begin{cases}\frac{\sqrt{1+4 p^{2}(1-p)^{2}}+\left(p^{2}+(1-p)^{2}\right)}{4 p(1-p)} & \text { if } p \in(0,1) \\ \frac{1}{|2 p-1|-1} & \text { if } p \in \mathbb{R} \backslash[0,1]\end{cases}
$$

Remark 2.6. Applying the above result for $p \in\left\{-\frac{1}{2}, \frac{1}{2}\right\}$ the optimal constant obtained by our method is equal to $\frac{1+\sqrt{5}}{2}$. Observe that it is smaller than the constant in $[12$, Th. 1].

In the case $p \in(0,1) \Longleftrightarrow p^{2}+(1-p)^{2}<1$ our estimation $M$ is smaller than the estimate $\frac{1}{1-p^{2}-(1-p)^{2}}$ obtained for the equation of the $p$-Wright affine function in [4, Th. 2. 1].

In the same manner we can study other cases in order to get the optimal constants.

\subsection{Linear equation}

Consider the linear equation

$$
f(a x+b y)=A f(x)+B f(y), \quad x, y \in X .
$$

We look for solutions $c_{1}, c_{2}$ of subsystems of the system of linear equations

$$
\left[\begin{array}{ll}
a & b \\
1 & 0 \\
0 & 1
\end{array}\right]\left[\begin{array}{l}
c_{1} \\
c_{2}
\end{array}\right]=\left[\begin{array}{l}
1 \\
1 \\
1
\end{array}\right]
$$


Thus, we have the following possibilities:

(1) $c_{1}=c_{2}=1, a+b=1$

(2) $c_{1}=c_{2}=1, a+b \neq 1$

(3) $c_{1}=1, c_{2}=\frac{1-a}{b} \neq 1, b \neq 0$

(3') $c_{2}=1, c_{1}=\frac{1-b}{a} \neq 1, a \neq 0$

(4) $c_{1}=c \neq 1, c_{2}=\frac{1-a c}{b} \neq 1, b \neq 0$

(4') $c_{2}=c \neq 1, c_{1}=\frac{1-b c}{a} \neq 1, a \neq 0$

(5) $c_{1}=1, c_{2}=c \neq 1, a+b c \neq 1$

(5') $c_{2}=1, c_{1}=c \neq 1, a c+b \neq 1$.

Therefore we obtain the results corresponding to each of the cases (number of each case is indicated in parentheses).

Theorem 2.7 (1). Let $Y$ be a Banach space, $a+b=1, A+B \neq 1, \theta: X^{2} \rightarrow \mathbb{R}_{+}$. If $g: X \rightarrow Y$, satisfies

$$
\|g(a x+b y)-A g(x)-B g(y)\| \leq \theta(x, y), \quad x, y \in X,
$$

then there exists a unique solution $G: X \rightarrow Y$ of (2.4) such that

$$
\|g(x)-G(x)\| \leq \frac{\theta(x, x)}{|1-A-B|}, \quad x \in X .
$$

Moreover $G$ is a unique solution of (2.4) such that there exists a constant $K \in(0, \infty)$ with

$$
\|g(x)-G(x)\| \leq K \theta(x, x), \quad x \in X .
$$

Theorem 2.8 (2). Let $Y$ be a Banach space, $a+b \neq 1, g: X \rightarrow Y, \theta: X^{2} \rightarrow \mathbb{R}_{+}$ satisfy (2.5). Assume that there exists $\omega \in \mathbb{R}_{+}$such that $\omega<|A+B|$ and

$$
\theta((a+b) x,(a+b) y) \leq \omega \theta(x, y), \quad x, y \in X .
$$

Then there exists a unique solution $G: X \rightarrow Y$ of (2.4) such that

$$
\|g(x)-G(x)\| \leq \frac{\theta(x, x)}{|A+B|-\omega}, \quad x \in X .
$$

Proof. Applying Theorem 2.1 for $c_{1}=c_{2}=1, I=\{2,3\}$ we obtain our claim.

Theorem 2.9 (3). Let $Y$ be a Banach space, $a+b \neq 1, b \neq 0, g: X \rightarrow Y$, $\theta: X^{2} \rightarrow \mathbb{R}_{+}$satisfy (2.5). Assume that there exists $\omega \in \mathbb{R}_{+}$such that $|B| \omega<$ $|1-A|$ and

$$
\theta\left(\frac{1-a}{b} x, \frac{1-a}{b} y\right) \leq \omega \theta(x, y) .
$$

Then there exists a unique solution $G: X \rightarrow Y$ of (2.4) such that

$$
\|g(x)-G(x)\| \leq \frac{\theta\left(x, \frac{1-a}{b} x\right)}{|1-A|-|B| \omega}, \quad x \in X .
$$


Theorem 2.10 (4). Let $Y$ be a Banach space, $a c+b \neq 1, b \neq 0, c \neq 1, g: X \rightarrow$ $Y, \theta: X^{2} \rightarrow \mathbb{R}_{+}$satisfy (2.5). Assume that there exist $\omega(c), \omega\left(\frac{1-a c}{b}\right) \in \mathbb{R}_{+}$ such that $|A| \omega(c)+|B| \omega\left(\frac{1-a c}{b}\right)<1$ and

$$
\theta(\beta x, \beta y) \leq \omega(\beta) \theta(x, y), \quad \beta \in\left\{c, \frac{1-a c}{b}\right\} .
$$

Then there exists a unique solution $G: X \rightarrow Y$ of (2.4) such that

$$
\|g(x)-G(x)\| \leq \frac{\theta\left(c x, \frac{1-a c}{b} x\right)}{1-|A| \omega(c)-|B| \omega\left(\frac{1-a c}{b}\right)}, \quad x \in X .
$$

Theorem 2.11 (5). Let $Y$ be a Banach space, $a+b c \neq 1, c \neq 1, g: X \rightarrow Y$, $\theta: X^{2} \rightarrow \mathbb{R}_{+}$satisfy (2.5). Assume that there exist $\omega(a+b c), \omega(c) \in \mathbb{R}_{+}$such that $\omega(a+b c)+|B| \omega(c)<|A|$ and

$$
\theta(\beta x, \beta y) \leq \omega(\beta) \theta(x, y), \quad \beta \in\{c, a+b c\} .
$$

Then there exists a unique solution $G: X \rightarrow Y$ of (2.4) such that

$$
\|g(x)-G(x)\| \leq \frac{\theta(x, c x)}{|A|-\omega(a+b c)-|B| \omega(c)}, \quad x \in X .
$$

Note that interchanging $a$ and $b, A$ and $B$ in the above three theorems, we obtain results for the cases $\left(3^{\prime}\right)-\left(5^{\prime}\right)$.

According to Remark 2.3, in the case $\theta(x, y):=\varepsilon$ we can take $\omega \equiv 1$. Consequently, combining all the above results in this case we deduce that the linear equation is stable and the bounding constant is the smallest one obtained by our method.

Theorem 2.12. Let $\mathbb{F}=\mathbb{R}, a+b=1, A+B \neq 1$. If $g: X \rightarrow Y$ satisfies

$$
\|g(a x+b y)-A g(x)-B g(y)\| \leq \varepsilon, \quad x, y \in X,
$$

then the linear equation (2.4) is stable. Namely, there exists its unique solution $G: X \rightarrow Y$ such that

$$
\|g(x)-G(x)\| \leq \frac{\varepsilon}{|A+B-1|}, \quad x \in X .
$$

Proof. Take $a, b, A, B \in \mathbb{R}$ such that $a+b=1$ and $A+B \neq 1$. Then at least the condition (1) holds, the conditions (2), (3), (3') are not satisfied.

Define constants

$$
\begin{array}{ll}
M_{1}=|A+B-1|, \quad & M_{4}=M_{4^{\prime}}=1-|A|-|B|, \\
M_{5}=|A|-1-|B|, & M_{5^{\prime}}=|B|-1-|A| .
\end{array}
$$

If $M_{i}>0$ for some $i \in\left\{1,4,5,5^{\prime}\right\}$, then there exists a unique soluton $G_{i}$ : $X \rightarrow Y$ of (2.4) such that

$$
\left\|g(x)-G_{i}(x)\right\| \leq \frac{\varepsilon}{M_{i}}, \quad x \in X,
$$

see Theorems 2.7, 2.10, 2.11 and analogons of the last two ones. 
It is easy to verify that $M_{1}=\max \left\{M_{1}, M_{4}, M_{5}, M_{5^{\prime}}\right\}$, therefore $\frac{1}{M_{1}}$ is the smallest bound obtained using linear substitutions. Moreover, according to the second assertion of Theorem 2.7 the solution $G_{1}$ is a unique one satisfying the inequality

$$
\|g(x)-G(x)\| \leq K \varepsilon, \quad x \in X
$$

with any constant $K \in \mathbb{R}$, which completes the proof.

The same, but more complicated reasoning, can be drawn for the case $a+b \neq 1$. To state our result in this case, define sets

$$
\begin{aligned}
P(3):=\left\{(A, B) \in \mathbb{R}^{2}:\right. & (B \geq-1 \wedge 1+A \leq B<1-A) \vee \\
& (-1 \leq B \leq 0 \wedge A-1<B \leq A) \vee(A+B<1 \wedge A \geq 0 \wedge B \geq 0)\}, \\
P\left(3^{\prime}\right):=\left\{(A, B) \in \mathbb{R}^{2}:\right. & (A \geq-1 \wedge 1+B \leq A<1-B) \vee \\
& (-1 \leq A \leq 0 \wedge B-1<A \leq B) \vee(A+B<1 \wedge A \geq 0 \wedge B \geq 0)\}, \\
P:=\left\{(A, B) \in \mathbb{R}^{2}:\right. & A+B=1\} .
\end{aligned}
$$

Theorem 2.13 (The case $\theta \equiv \varepsilon$ ). Let $\mathbb{F}=\mathbb{R}, a+b \neq 1, A+B \neq 1$. If $g: X \rightarrow Y$ satisfies

$$
\|g(a x+b y)-A g(x)-B g(y)\| \leq \varepsilon, \quad x, y \in X,
$$

then the linear equation is stable. Namely, there exists its unique solution $G$ : $X \rightarrow Y$ such that

$$
\|g(x)-G(x)\| \leq \frac{\varepsilon}{M(A, B)}, \quad x \in X,
$$

where

$$
M(A, B)=\left\{\begin{array}{ll}
|1-A|-|B| & \text { if }(A, B) \in P(3) \\
|1-B|-|A| & \text { if }(A, B) \in P\left(3^{\prime}\right) \\
|A+B|-1 & \text { if }(A, B) \in \mathbb{R}^{2} \backslash\left(P(3) \cup P\left(3^{\prime}\right) \cup P\right)
\end{array} .\right.
$$

In the case $A+B=1$ our criterion does not determine the stability of the equation (2.4) for the constant control function.

Remark 2.14. Among a lot of consequences of Theorems 2.7-2.13, we have the following results for Cauchy's equation (the case $a=b=A=B=1$ ).

Applying Theorem 2.13 for $\theta(x, y):=\varepsilon$ and $\omega=1$ we have the well known stability result proved by Hyers in [9]. Observe that the constant $M(A, B)=$ $|A+B|-1$ obtained by our method is optimal.

Theorem 2.8 used for the case $\theta(x, y):=C\left(\|x\|^{p}+\|y\|^{p}\right), \omega=|a+b|^{p}$ leads to Aoki's outcome from [1].

Setting in Theorem $2.10 c=\frac{1}{2}$ and $\theta(x, y):=C\left(\|x\|^{p}+\|y\|^{p}\right), \omega(c):=|c|^{p}$, where $C \geq 0, p>1$ we get the stability result for Cauchy's equation proved by Gajda (see [7]). 
It was proved in [2] that the estimation $\frac{C\|x\|^{p}}{\left|2^{p-1}-1\right|}$ is the optimum for $p \geq 0$ and $p \neq 1$ in the general case.

\subsection{Quadratic equation}

Consider the quadratic equation

$$
f(x+y)+f(x-y)=2 f(x)+2 f(y), \quad x, y \in X,
$$

which is a particular case of (1.1), with $m=4, n=2, A_{1}=A_{2}=1, A_{3}=$ $A_{4}=-2, a_{12}=a_{42}=a_{i 1}=1$ for $i=1,2,3, a_{32}=a_{41}=0, a_{22}=-1$. Applying Theorem 2.1 for $c_{1}=c_{2}=1, I=\{3,4\}$ we obtain the following stability result for this equation.

Theorem 2.15. Let $Y$ be a Banach space, $g: X \rightarrow Y, \theta: X^{2} \rightarrow \mathbb{R}_{+}$satisfy

$$
\|g(x+y)+g(x-y)-2 g(x)-2 g(y)\| \leq \theta(x, y), \quad x, y \in X .
$$

Assume that there exist $\omega(2), \omega(0) \in \mathbb{R}_{+}$such that $\omega(2)+\omega(0)<4$ and

$$
\theta(c x, c y) \leq \omega(c) \theta(x, y), \quad c \in\{2,0\} .
$$

Then there exists a unique solution $G: X \rightarrow Y$ of (2.6) such that

$$
\|g(x)-G(x)\| \leq \frac{\theta(x, x)}{4-\omega(2)-\omega(0)}, \quad x \in X .
$$

Moreover $G$ is a unique solution of (3.2) such that there exists a constant $K \in(0, \infty)$ with

$$
\|g(x)-G(x)\| \leq K \theta(x, x), \quad x \in X .
$$

Remark 2.16. Applying the above theorem for $\theta(x, y):=\varepsilon, \omega(c):=1$ we obtain the known result of Skof. For the functions $\theta(x, y):=C\left(\|x\|^{p}+\|y\|^{p}\right)$, $\omega(c)=|c|^{p}, p<2$ we obtain the estimation $\frac{C\|x\|^{p}}{4-2^{p}}$.

\section{Proof of the main result}

In the sequel, $\mathcal{T}$ and $\Lambda$ stand for maps of the forms

$$
\begin{aligned}
& (\mathcal{T} \xi)(x):=\sum_{i=1}^{k} \alpha_{i} \xi\left(\beta_{i} x\right), \quad \xi \in Y^{X}, x \in X, \\
& (\Lambda \delta)(x):=\sum_{i=1}^{k}\left|\alpha_{i}\right| \delta\left(\beta_{i} x\right), \quad \delta \in \mathbb{R}_{+}{ }^{X}, x \in X,
\end{aligned}
$$

for some $\alpha_{1}, \ldots, \alpha_{k}, \beta_{1}, \ldots, \beta_{k} \in \mathbb{F}, k \in \mathbb{N}$.

Moreover we have the following lemmas, which will be needed to obtain our main result. 
Lemma 3.1. Let $X$ be a linear space over $\mathbb{F}, \varepsilon \in \mathbb{R}_{+}{ }^{X}$ and let $\Lambda: \mathbb{R}_{+}{ }^{X} \rightarrow \mathbb{R}_{+}{ }^{X}$ be given by (3.2). If there exist $\omega_{1}, \ldots, \omega_{k} \in \mathbb{R}_{+}$such that

$$
\varepsilon\left(\beta_{i} x\right) \leq \omega_{i} \varepsilon(x), \quad i \in\{1, \ldots, k\}, x \in X,
$$

then

$$
\sum_{n=0}^{\infty}\left(\Lambda^{n} \varepsilon\right)(x) \leq \varepsilon(x) \sum_{n=0}^{\infty}\left(\sum_{i=1}^{k}\left|\alpha_{i}\right| \omega_{i}\right)^{n} .
$$

Moreover if $\gamma:=\sum_{i=1}^{k}\left|\alpha_{i}\right| \omega_{i}<1$, then $\sum_{n=0}^{\infty}\left(\Lambda^{n} \varepsilon\right)(x) \leq \frac{\varepsilon(x)}{1-\gamma}$.

Proof. Denote $\gamma:=\sum_{i=1}^{k}\left|\alpha_{i}\right| \omega_{i}$. We prove that for every $x \in X$ and $l \in \mathbb{N}_{0}$ (nonnegative integers)

$$
\Lambda^{l} \varepsilon(x) \leq \varepsilon(x) \gamma^{l} .
$$

Obviously, the above inequality is fulfilled for $l=0$. Take $x \in X$ and $l \in \mathbb{N}_{0}$ and assume (3.4). Thus

$$
\begin{aligned}
\left(\Lambda^{l+1} \varepsilon\right)(x) & =\Lambda\left(\Lambda^{l} \varepsilon\right)(x)=\sum_{i=1}^{k}\left|\alpha_{i}\right|\left(\Lambda^{l} \varepsilon\right)\left(\beta_{i} x\right) \leq \sum_{i=1}^{k}\left|\alpha_{i}\right| \varepsilon\left(\beta_{i} x\right) \gamma^{l} \\
& \leq \gamma^{l}\left(\sum_{i=1}^{k}\left|\alpha_{i}\right| \omega_{i}\right) \varepsilon(x)=\varepsilon(x) \gamma^{l+1}
\end{aligned}
$$

and by induction the proof of the first assertion is completed. The second one is a consequence of the convergence of the power series.

Observe that operators (3.1) and (3.2) satisfy the assumptions of Theorem 1 in [3], therefore applying this version of the fixed point theorem and the above lemma we have the following result.

Lemma 3.2. Let $Y$ be a Banach space, $\varepsilon \in \mathbb{R}_{+}{ }^{X}$ and let $\mathcal{T}: Y^{X} \rightarrow Y^{X}$ be given by (3.1). Assume that there exist $\omega_{1}, \ldots, \omega_{k} \in \mathbb{R}_{+}$such that $\gamma:=\sum_{i=1}^{k}\left|\alpha_{i}\right| \omega_{i}<$ 1 and the condition (3.3) holds. If $g: X \rightarrow Y$ satisfies the inequality

$$
\|(\mathcal{T} g)(x)-g(x)\| \leq \varepsilon(x), \quad x \in X,
$$

then there exists a unique fixed point $G$ of $\mathcal{T}$ with

$$
\|g(x)-G(x)\| \leq \frac{\varepsilon(x)}{1-\gamma}, \quad x \in X .
$$

Moreover $G(x):=\lim _{n \rightarrow \infty}\left(\mathcal{T}^{n} g\right)(x), x \in X$.

Lemma 3.3. Assume that $Y$ is a Banach space, $\theta: X^{n} \rightarrow \mathbb{R}_{+}$and let $\mathcal{T}$ : $Y^{X} \rightarrow Y^{X}$ be given by (3.1). Assume that there exist $\omega_{1}, \ldots \omega_{k} \in \mathbb{R}_{+}$such that $\sum_{i=1}^{k}\left|\alpha_{i}\right| \omega_{i}<1$, and

$$
\theta\left(\beta_{i} x_{1}, \ldots, \beta_{i} x_{n}\right) \leq \omega_{i} \theta\left(x_{1}, \ldots, x_{n}\right) \quad i \in\{1, \ldots, k\}, x_{1}, \ldots, x_{n} \in X .
$$


If $g: X \rightarrow Y$ satisfies the inequality

$$
\left\|\sum_{i=1}^{m} A_{i} g\left(\sum_{j=1}^{n} a_{i j} x_{j}\right)\right\| \leq \theta\left(x_{1}, \ldots, x_{n}\right), \quad x_{1}, \ldots, x_{n} \in X,
$$

and for every $x \in X$ there exists $G(x):=\lim _{n \rightarrow \infty} \mathcal{T}^{n} g(x)$, then $G: X \rightarrow Y$ is a solution to (1.1) (with $A=0)$.

Proof. Denote

$$
\Phi(\xi)\left(x_{1}, \ldots, x_{n}\right)=\sum_{i=1}^{m} A_{i} \xi\left(\sum_{j=1}^{n} a_{i j} x_{j}\right), \quad \xi \in Y^{X}, x_{1}, \ldots, x_{n} \in X .
$$

Let $\gamma:=\sum_{j=1}^{k}\left|\alpha_{j}\right| \omega_{j}$. We prove that for every $x_{1}, \ldots, x_{n} \in X$ and $l \in \mathbb{N}_{0}$

$$
\left\|\Phi\left(\mathcal{T}^{l} g\right)\left(x_{1}, \ldots, x_{n}\right)\right\| \leq \gamma^{l} \theta\left(x_{1}, \ldots, x_{n}\right) .
$$

Clearly, the case $l=0$ is just (3.7). Next, fix $l \in \mathbb{N}_{0}$ and assume that (3.8) holds for every $x_{1}, \ldots, x_{n} \in X$. Then for every $x_{1}, \ldots, x_{n} \in X$

$$
\begin{aligned}
& \Phi\left(\mathcal{T}^{l+1} g\right)\left(x_{1}, \ldots, x_{n}\right)=\sum_{i=1}^{m} A_{i}\left(\mathcal{T}^{l+1} g\right)\left(\sum_{j=1}^{n} a_{i j} x_{j}\right)=\sum_{i=1}^{m} A_{i} \mathcal{T}\left(\mathcal{T}^{l} g\right)\left(\sum_{j=1}^{n} a_{i j} x_{j}\right) \\
& =\sum_{i=1}^{m} A_{i} \sum_{p=1}^{k} \alpha_{p}\left(\mathcal{T}^{l} g\right)\left(\beta_{p} \sum_{j=1}^{n} a_{i j} x_{j}\right)=\sum_{p=1}^{k} \alpha_{p}\left[\sum_{i=1}^{m} A_{i}\left(\mathcal{T}^{l} g\right)\left(\sum_{j=1}^{n} a_{i j}\left(\beta_{p} x_{j}\right)\right)\right] \\
& =\sum_{p=1}^{k} \alpha_{p} \Phi\left(\mathcal{T}^{l} g\right)\left(\beta_{p} x_{1}, \ldots, \beta_{p} x_{n}\right) .
\end{aligned}
$$

Consequently, applying the inductive assumption and (3.6)

$$
\begin{aligned}
\| & \Phi\left(\mathcal{T}^{l+1} g\right)\left(x_{1}, \ldots, x_{n}\right)\left\|\leq \sum_{p=1}^{k}\left|\alpha_{p}\right|\right\| \Phi\left(\mathcal{T}^{l} g\right)\left(\beta_{p} x_{1}, \ldots, \beta_{p} x_{n}\right) \| \\
& \leq \sum_{p=1}^{k}\left|\alpha_{p}\right| \gamma^{l} \theta\left(\beta_{p} x_{1}, \ldots, \beta_{p} x_{n}\right) \leq \sum_{p=1}^{k}\left|\alpha_{p}\right| \gamma^{l} \omega_{p} \theta\left(x_{1}, \ldots, x_{n}\right) \\
& =\gamma^{l+1} \theta\left(x_{1}, \ldots, x_{n}\right) .
\end{aligned}
$$

Thus, by induction we have shown that (3.8) holds for $x_{1}, \ldots, x_{n} \in X$, $l \in \mathbb{N}_{0}$.

Observe that

$$
\lim _{l \rightarrow \infty} \Phi\left(\mathcal{T}^{l} g\right)\left(x_{1}, \ldots, x_{n}\right)=\Phi\left(\lim _{l \rightarrow \infty} \mathcal{T}^{l} g\right)\left(x_{1}, \ldots, x_{n}\right) .
$$

Since by our assumptions $\gamma=\sum_{i=1}^{k}\left|\alpha_{i}\right| \omega_{i}<1$, letting $l \rightarrow \infty$ in (3.8), we obtain that 


$$
\Phi(G)\left(x_{1}, \ldots, x_{n}\right)=0, \quad x_{1}, \ldots, x_{n} \in X
$$

Now we are in a position to prove Theorem 2.1

Proof. Assume that $\emptyset \neq I \subset\{1, \ldots, m\}$ and $c_{1}, \ldots, c_{n} \in \mathbb{F}, \omega_{1}, \ldots, \omega_{n} \in \mathbb{F}$ such that assumptions (i)-(iii) hold. Note that $A_{I}:=\sum_{i \in I} A_{i} \neq 0$ by (ii). The proof will be divided into 2 steps.

First assume that $A=0$. Substituting $x_{j}=c_{j} x, j \in\{1, \ldots, n\}$ in (2.1) we have

$$
\|g(x)-\mathcal{T} g(x)\| \leq \frac{\theta\left(c_{1} x, \ldots, c_{n} x\right)}{\left|A_{I}\right|}, \quad x \in X,
$$

where for every $\xi \in Y^{X}, x \in X$

$$
\mathcal{T} \xi(x):=\left\{\begin{array}{ll}
\sum_{i \notin I} \frac{-A_{i}}{A_{I}} \xi\left(\sum_{j=1}^{n} a_{i j} c_{j} x\right) & \text { if } I \neq\{1, \ldots, m\} \\
0 & \text { if } I=\{1, \ldots, m\}
\end{array} .\right.
$$

Define moreover $\varepsilon(x):=\frac{\theta\left(c_{1} x, \ldots, c_{n} x\right)}{\left|A_{I}\right|}$ for $x \in X$ and put $\gamma:=\sum_{i \notin I}\left|\frac{A_{i}}{A_{I}}\right| \omega_{i}$, according to our convention that the value of an empty sum is zero.

Observe that all the assumptions of Lemma 3.2 are fulfilled. Hence there exists a unique fixed point $G: X \rightarrow Y$ of $\mathcal{T}$ such that

$$
\|g(x)-G(x)\| \leq \frac{\varepsilon(x)}{1-\gamma}=\frac{\theta\left(c_{1} x, \ldots, c_{n} x\right)}{\left|A_{I}\right|-\sum_{i \notin I}\left|A_{i}\right| \omega_{i}}, \quad x \in X,
$$

and $G(x)=\lim _{n \rightarrow \infty}\left(\mathcal{T}^{n} g\right)(x)$ for $x \in X$. By Lemma 3.3, it is a solution of the equation (1.1) (with $A=0$ ).

Suppose now that there exist $B>0$ and $H: X \rightarrow Y$ a solution of (1.1) such that (2.3) is satisfied. By the triangle inequality and (3.9)

$$
\begin{aligned}
\|G(x)-H(x)\| & \leq \frac{\varepsilon(x)}{1-\gamma}+B \theta\left(c_{1} x, \ldots, c_{n} x\right)=\frac{\varepsilon(x)}{1-\gamma}+B\left|A_{I}\right| \varepsilon(x) \\
& =\left(1+B\left|A_{I}\right|(1-\gamma)\right) \varepsilon(x) \frac{1}{1-\gamma} \\
& =\left(1+B A_{I}(1-\gamma)\right) \varepsilon(x) \sum_{p=0}^{\infty} \gamma^{p}, \quad x \in X
\end{aligned}
$$

Let $C:=1+B\left|A_{I}\right|(1-\gamma)$. We show that for all $l \in \mathbb{N}_{0}$ and $x \in X$

$$
\|G(x)-H(x)\| \leq C \varepsilon(x) \sum_{p=l}^{\infty} \gamma^{p} .
$$

The case $l=0$ is exactly (3.10). So fix $x \in X$ and assume that (3.11) holds for $l \in \mathbb{N}_{0}$, and we will prove it for $l+1$. Observe that for every $x \in X$ the 
equation (1.1) is satisfied for $c_{1} x, \ldots, c_{n} x$, therefore

$$
\mathcal{T} H(x)=\sum_{i \notin I} \frac{-A_{i}}{A_{I}} H\left(\sum_{j=1}^{n} a_{i j} c_{j} x\right)=\sum_{i \in I} \frac{A_{i}}{A_{I}} H\left(\sum_{j=1}^{n} a_{i j} c_{j} x\right)=H(x)
$$

and $H$ is a fixed point of $\mathcal{T}$. Consequently

$$
\begin{aligned}
& \|G(x)-H(x)\|=\|\mathcal{T} G(x)-\mathcal{T} H(x)\| \\
& =\left\|\sum_{i \notin I} \frac{-A_{i}}{A_{I}} G\left(\sum_{j=1}^{n} a_{i j} c_{j} x\right)-\sum_{i \notin I} \frac{-A_{i}}{A_{I}} H\left(\sum_{j=1}^{n} a_{i j} c_{j} x\right)\right\| \\
& \leq \sum_{i \notin I}\left|\frac{-A_{i}}{A_{I}}\right| \| G\left(\sum_{j=1}^{n} a_{i j} c_{j} x\right)-H\left(\sum_{j=1}^{n} a_{i j} c_{j} x\right) \mid \\
& \leq \sum_{i \notin I}\left|\frac{-A_{i}}{A_{I}}\right| C \varepsilon\left(\sum_{j=1}^{n} a_{i j} c_{j} x\right) \sum_{p=l}^{\infty} \gamma^{p} \leq C \sum_{i \notin I}\left|\frac{-A_{i}}{A_{I}}\right| \omega_{i} \varepsilon(x) \sum_{p=l}^{\infty} \gamma^{p} \\
& =C \varepsilon(x) \sum_{p=l+1}^{\infty} \gamma^{p} .
\end{aligned}
$$

Letting $l \rightarrow \infty$ in (3.11) we get $G=H$ and the proof in the first case is complete.

If $A \neq 0$ and $\sum_{i=1}^{m} A_{i} \neq 0$ define $f(x):=g(x)+\frac{A}{\sum_{i=1}^{m} A_{i}}, x \in X$. By

$$
\left\|\sum_{i=1}^{m} A_{i} f\left(\sum_{j=1}^{n} a_{i j} x_{j}\right)\right\| \leq \theta\left(x_{1}, \ldots, x_{n}\right), \quad x_{1}, \ldots, x_{n} \in X
$$

and consequently, according to our previous considerations, there exists a unique function $F: X \rightarrow Y$ such that

$$
\|f(x)-F(x)\| \leq \frac{\theta\left(c_{1} x, \ldots, c_{n} x\right)}{\left|A_{I}\right|-\sum_{i \notin I}\left|A_{i}\right| \omega_{i}}, \quad x \in X .
$$

Obviously $G(x):=F(x)-\frac{A}{\sum_{i=1}^{m} A_{i}}, x \in X$ is the desired function.

Open Access. This article is distributed under the terms of the Creative Commons Attribution License which permits any use, distribution, and reproduction in any medium, provided the original author(s) and the source are credited.

\section{References}

[1] Aoki, T.: On the stability of the linear transformation in Banach spaces. J. Math. Soc. Japan 2, 64-66 (1950) 
[2] Brzdęk, J.: A note on stability of additive mappings. In: Rassias, Th.M., Tabor, J. (eds.) Stability of Mappings of Hyers- Ulam Type, pp. 19-22. Hadronic Press, Inc., Florida (1994)

[3] Brzdęk, J., Chudziak, J., Páles, Zs.: A fixed point approach to stability of functional equations. Nonlinear Anal. 74, 6728-6732 (2011)

[4] Brzdęk, J.: Stability of the equation of the $p$-Wright affine functions. Aequationes Math. 85, 497-503 (2013)

[5] Brzdęk J.: Ciepliński, hyperstability and superstability. Abstr. Appl. Anal. (2013). doi:10.1155/2013/401756

[6] Forti, G.-L.: Elementary remarks on Ulam-Hyers stability of linear functional equations. J. Math. Anal. Appl. 328, 109-118 (2007)

[7] Gajda, Z.: On stability of additive mappings. Int. J. Math. Sci. 14, 431-434 (1991)

[8] Gilányi, A., Páles, Zs.: On Dinghas-type derivatives and convex functions of higher order. Real Anal. Exchange, 27, 485-493 (2001/2002)

[9] Hyers, D.H.: On the stability of the linear functional equation. Proc. Nat. Acad. Sci. USA 27, 222-224 (1941)

[10] Hyers, D.H., Isac, G., Rassias, Th. M.: Stability of Functional Equations in Several Variables. Birkhäuser, Boston (1998)

[11] Jung S.-M.: Hyers-Ulam-Rassias Stability of Functional Equations in Nonlinear Analysis. Springer Optimization and Its Applications, vol. 48, Springer, New York-DordrechtHeidelberg-London (2011)

[12] Jung, S.-M.: Hyers-Ulam-Rassias stability of Jensen's equation and its application. Proc. Am. Math. Soc. 126(11), 3137-3143 (1998)

[13] Lajkó, K.: On a functional equation of Alsina and García-Roig. Publ. Math. Debrecen 52, 507-515 (1998)

[14] Maksa, Gy., Nikodem, K., Páles, Zs.: Results on $t$-Wright convexity. C. R. Math. Rep. Acad. Sci. Canada 13, 274-278 (1991)

[15] Paneah, B.: A new approach to the stability of linear functional operators. Aequationes Math. 78, 45-61 (2009)

[16] Rassias, M.: On a modified Hyers-Ulam sequence. J. Math. Anal. Appl. 158, 106$113(1991)$

[17] Skof, F.: Proprieta locali e approssimazione di operatori. Rend. Sem. Mat. Fis. Milano 53, 113-129 (1983)

Anna Bahyrycz and Jolanta Olko

Institute of Mathematics

Pedagogical University

Podchorążych 2

30-084 Kraków

Poland

e-mail: bah@up.krakow.pl

e-mail: jolko@up.krakow.pl

Received: July 29, 2014 